\title{
SELETIVIDADE E EFICÁCIA DE MISTURAS DE HERBICIDAS DO GRUPO DAS IMIDAZOLINONAS EM MILHO TOLERANTE
}

\author{
Roberto C. Pereira ${ }^{1}$ e Ricardo Carmona ${ }^{1}$
}

${ }^{1}$ Universidade de Brasília. Caixa Postal 04508. Brasília, DF 70910-970

\section{RESUMO}

Testou-se o comportamento de misturas de herbicidas do grupo das imidazolinonas, aplicadas em pré-emergência e pós-emergência, no controle de Richardia brasiliensis, Acanthospermum australe, Brachiaria decumbens e Spermacoce latifolia em um híbrido de milho tolerante a este grupo de herbicidas. Em pré-emergência Foram avaliadas as misturas imazethapyr/imazapyr $(79+26,90+30,70+35$ e $80+40 \mathrm{~g} / \mathrm{ha})$, imazethapyr/imazapic $(60+30,70+35 \mathrm{e} 80+40 \mathrm{~g} / \mathrm{ha}) \mathrm{e}$ imazapic/imazapyr $(68+22,70+35$ e $79+26 \mathrm{~g} / \mathrm{ha})$ usando-se como padrão de comparação a mistura atrazine/metolachlor $(3250 \mathrm{~g} / \mathrm{ha})$. Em pós-emergência foram utilizadas as misturas imazethapyr/imazapyr $(57+18,68+22,79+26,50+25,60+30$ e $70+35 \mathrm{~g} / \mathrm{ha})$ e imazapic/imazapyr (40+20, 50+25, 60+30, 45+15, 57+18 e 68+22 g/ha), usando-se como padrão de comparação o herbicida nicosulfuron (60 g/ha). Além disto foram incluídas duas testemunhas sem herbicidas, na presença e ausência de capina. Concluiu-se que nenhuma mistura, independente da época de aplicação causou toxicidade à cultura do milho. Nas doses utilizadas, as misturas imazethapyr/imazapyr, imazapic/imazapyr e imazethapy/imazapic, aplicadas em pré-emergência, controlaram de forma excelente (superior a 90\%) o A. australe, R. brasiliensis e $S$. latifolia, sendo que o imazethapyr/ imazapic também controlou de forma excelente a $B$. decumbens. Nas aplicações em pós-emergência o controle excelente de $R$. brasiliensis e $A$. australe só foi obtido com a mistura imazapic/imazapyr, sendo o $A$. australe também controlado com imazethapyr/imazapyr. O controle de $B$. decumbens foi no máximo aceitável (80-89\%) nas aplicações em pós-emergência.

Palavras-chave: Acanthospermum australe, Brachiaria decumbens, Richardia brasiliensis, Spermacoce latifolia.

\section{ABSTRACT \\ Efficacy of mixtures of imidazolinone herbicides on tolerant mayze}

Mixtures of imidazolinone herbicides were applied in pre and postemergence on a hibrid of maize tolerant to this group, to control Richardia brasiliensis, Acanthospermum australe, Brachiaria decumbens and Spermacoce latifolia. Two experiments were conducted, one in each time of application. In pre-emergence the following mixtures were tested: imazethapyr/ imazapyr $(79+26,90+30,70+35$ and $80+40 \mathrm{~g} / \mathrm{ha})$, imazethapyr/imazapic $(60+30,70+35$ and $80+40 \mathrm{~g} / \mathrm{ha})$, imazapic/ imazapyr $(68+22,70+35$ and $79+26 \mathrm{~g} / \mathrm{ha})$, compared with atrazine/metolachlor $(3250 \mathrm{~g} / \mathrm{ha})$. The following mixtures were tested in postemergence: imazethapyr/imazapyr $(57++18,68+22,79+26,50+25,60+30$ and $70+35 \mathrm{~g} / \mathrm{ha})$, imazapic/imazapyr $(40+20,50+25,60+30,45+15,57+18$ and $68+22 \mathrm{~g} / \mathrm{ha})$, compared with nicosulfuron $(60 \mathrm{~g} / \mathrm{ha})$. Two other controls were also included on both experiments: presence or absence of hand weeding. No toxicity was observed on maize by any mixture, dose or time of application. The used doses of imazethapyr/imazapyr, imazapic/imazapyr and imazethapyr/imazapic, applied in pre-emergence, showed an excellent control of $A$. australe, $R$. brasiliensis and $S$. latifolia. Imazethapyr/imazapic also controlled $B$. decumbens. In postemergence application an excellent control of both $R$. brasiliensis and A. australe was only achieved with imazapic/imazapyr. Imazethapyr/imazapyr also controlled very well $A$. australe. The control of $B$. decumbens was acceptable in postemergence applications.

Key words: Acanthospermum australe, Brachiaria decumbens, Richardia brasiliensis, Spermacoce latifolia. 


\section{INTRODUÇÃO}

Os herbicidas imazethapyr, imazapyr, imazapic e outras imidazolinonas normalmente não são indicados para a cultura do milho. Chega-se a recomendar um período de 270 dias entre uma aplicação de imazaquin e o plantio de milho em sucessão ou rotação de culturas, para evitar problemas de resíduos.

Nos últimos anos, no entanto, têm sido criados híbridos de milho tolerantes ou resistentes às imidazolinonas. Essa resistência ou tolerância não é somente obtida pela inserção de gens, mas também, pela alta pressão de seleção por gens que ocorrem naturalmente. A incorporação de gens não melhora a atuação dos híbridos de milho com relação a outros parâmetros que não a tolerância às imidazolinonas e nenhuma semente de milho tolerante às imidazolinonas é melhor que sua base genética. No entanto, a possibilidade do uso de herbicidas do grupo dos imidazolinonas na cultura do milho oferecerá aos produtores um maior número de opções para manejo das plantas daninhas (Loux et al. 1997).

A mistura formulada imazethapyr/imazapyr foi desenvolvida especificamente para utilização em milho tolerante/resistente a herbicidas do grupo das imidazolinonas (American Cyanimid Company, 1997). Essa mistura pode ser aplicada em pós-emergência inicial sobre o milho, obtendo-se um grande espectro de controle das plantas daninhas, sem qualquer decréscimo na produtividade. Alguma injúria pode ocorrer na cultura, caracterizada por amarelecimento e necrose das folhas, sintomas que desaparecem quinze dias após a aplicação do herbicida (American Cyanamid Company, 1997).

O objetivo do trabalho foi avaliar a seletividade e a eficácia de misturas de herbicidas do grupo das imidazolinonas, aplicadas em pré-emergência e pós-emergência, no controle de plantas daninhas na cultura do milho, utilizando-se híbrido resistente/tolerante às imidazolinonas.

\section{MATERIAL E MÉTODOS}

Os experimentos foram conduzidos em Planaltina-DF, em cultura de milho híbrido IMI-Corn semeado em 12/12/ 97 no sistema convencional, utilizando-se o espaçamento de $0,90 \mathrm{~m}$ entre linhas, densidade de seis sementes por metro linear e profundidade de semeadura de $4-5 \mathrm{~cm}$. ((Embrapa, 1998).

O solo, de classe textural argilosa (45\% de argila), com 2,8\% de matéria orgânica, foi adubado com $400 \mathrm{~kg} / \mathrm{ha}$ da fórmula 4-15-30 (N-P-K). O delineamento experimental de ambos experimentos foi o de blocos casualizados repetidos 4 vezes em parcelas medindo $4 \mathrm{~m} \times 7 \mathrm{~m}$. Além das testemunhas, capinada e não capinada, foram mantidas faixas de testemunha auxiliar (sem capina) ao longo das parcelas, destinadas à análise comparativa de infestação e do estado fitossanitário da cultura. Num experimento as aplicações foram efetuadas em pré-emergência, constando de treze tratamentos. O outro constou de quinze tratamentos com aplicações em pós-emergência.

Os herbicidas utilizados pertencem ao grupo químico das imidazolinonas (imazethapyr, imazapyr e imazapic), sulfoniluréias (nicosulfuron), triazinas (atrazine) e acetanilidas (metolachlor). O produto utilizado nas misturas imazethapyr/ imazapyr e imazapic/imazapyr foi formulado em grânulos dispersíveis em água. Na mistura atrazine/metolachlor foi utilizado o Primestra SC e o nicosulfuron foi representado pelo Sanson 40 SC. Várias doses das misturas foram utilizadas nos experimentos de pré e pós-emergência (Tabela 1).

As aplicações em pré-emergência foram efetuadas no dia $15 / 12 / 97$, três dias após a semeadura, utilizando-se um pulverizador manual propelido a $\mathrm{C}_{2}$, equipado com uma barra de $1,5 \mathrm{~m}$ contendo quatro bicos leque tipo 110.03 , trabalhando a uma pressão de $2,1 \mathrm{~kg} / \mathrm{cm}^{2}$ e proporcionando um volume de calda de 250 1/ha. No experimento de pós-emergência inicial, a aplicação dos produtos foi realizada no dia $23 / 12 / 97$, onze dias após a semeadura, quando as plantas daninhas se encontravam no estádio de 3-4 folhas, utilizando-se a mesma tecnologia de aplicação do experimento em pré-emergência.

As condições ambientais no momento da aplicação no dia 15/12/97, entre 14:00 e 14:30 horas, foram de céu claro, umidade relativa de $49 \%$ e temperatura do ar de $28^{\circ}$ C, não tendo ocorrido precipitação pluvial nas 24 horas após a aplicação dos herbicidas. Nos dez dias anteriores à aplicação choveu $29,6 \mathrm{~mm}$ e nos dez dias posteriores $27,8 \mathrm{~mm}$. No dia 23/12/97, entre 17:00 e 18:00 horas, o céu estava claro, umidade relativa de $55 \%$ e temperatura do ar de $27^{\circ} \mathrm{C}$, não tendo ocorrido precipitação pluviométrica nas 24 horas após a aplicação dos herbicidas. Nos dez dias anteriores à aplicação choveu 29,6 mm e nos dez dias posteriores 29,2 mm.

As avaliações de fitotoxicidade foram realizadas aos 30 e 60 dias após os tratamentos (DAT), utilizando-se a escala da EWRC (European Weed Research Council). Segundo essa escala 1 = ausência de danos, 2 = sintoma muito leve, 3 = dano leve aceito na prática, 4 = forte clorose, sem efeito na produção $5=$ duvidoso, $6=$ prejuízo evidente, $7=$ prejuízo pesado na colheita, $8=$ prejuízo muito pesado e $9=$ perda total (Australian Weeds Committee, 1979).

As avaliações de eficácia foram realizadas aos 30 e 60 dias após os tratamentos (DAT) e na pré-colheita, utilizando-se a escala de eficácia de controle de Frans e Talbert (1977), na qual 100 significa controle total, 90-99 controle excelente, 80-89 controle aceitável, 70-79 controle não aceitável, 40-69 controle deficiente, 10-39 controle fraco e 0-9 nenhum efeito). 
Tabela 1. Produtos utilizados nos dois experimentos (pré e pós-emergência), doses e concentração. Planaltina-DF, 1997.

\begin{tabular}{|c|c|c|c|c|}
\hline \multirow{2}{*}{ Experimento } & \multicolumn{2}{|c|}{ Produto técnico } & \multicolumn{2}{|c|}{ Produto formulado } \\
\hline & Nome & Dose (g/ha) & Concentração & Dose (/ha) \\
\hline \multirow{11}{*}{ Pré-emergência } & \multirow{4}{*}{ imazethapyr/imazapyr } & $79+26$ & \multirow{4}{*}{$700 \mathrm{~g} / \mathrm{kg}$} & $150 \mathrm{~g}$ \\
\hline & & $90+30$ & & $170 \mathrm{~g}$ \\
\hline & & $70+35$ & & $150 \mathrm{~g}$ \\
\hline & & $80+40$ & & $170 \mathrm{~g}$ \\
\hline & \multirow{3}{*}{ Imazethapyr/ imazapic } & $70+35$ & \multirow{3}{*}{$700 \mathrm{~g} / \mathrm{kg}$} & $150 \mathrm{~g}$ \\
\hline & & $80+40$ & & $170 \mathrm{~g}$ \\
\hline & & $60+30$ & & $130 \mathrm{~g}$ \\
\hline & \multirow{3}{*}{ imazapic/ imazapyr } & $70+35$ & \multirow{3}{*}{$700 \mathrm{~g} / \mathrm{kg}$} & $150 \mathrm{~g}$ \\
\hline & & $68+22$ & & $130 \mathrm{~g}$ \\
\hline & & $79+26$ & & $150 \mathrm{~g}$ \\
\hline & Atrazine/ metolachlor & $1300+1950$ & $500 \mathrm{~g} / 1$ & 6,51 \\
\hline \multirow{13}{*}{ Pós-emergência } & \multirow{6}{*}{ imazethapyr/ imazapyr } & $57+18$ & \multirow{3}{*}{$525+175 \mathrm{~g} / \mathrm{kg}$} & $110 \mathrm{~g}$ \\
\hline & & $68+22$ & & $130 \mathrm{~g}$ \\
\hline & & $79+26$ & & $150 \mathrm{~g}$ \\
\hline & & $50+25$ & \multirow{3}{*}{$467+233 \mathrm{~g} / \mathrm{kg}$} & $110 \mathrm{~g}$ \\
\hline & & $60+30$ & & $130 \mathrm{~g}$ \\
\hline & & $70+35$ & & $150 \mathrm{~g}$ \\
\hline & \multirow{6}{*}{ imazapic/imazapyr } & $40+20$ & \multirow{3}{*}{$467+251 \mathrm{~g} / \mathrm{kg}$} & $110 \mathrm{~g}$ \\
\hline & & $50+25$ & & $130 \mathrm{~g}$ \\
\hline & & $60+30$ & & $150 \mathrm{~g}$ \\
\hline & & $45+15$ & \multirow{3}{*}{$525+75 \mathrm{~g} / \mathrm{kg}$} & $110 \mathrm{~g}$ \\
\hline & & $57+18$ & & $130 \mathrm{~g}$ \\
\hline & & $68+22$ & & $150 \mathrm{~g}$ \\
\hline & nicosulfuron & 50 & $40 \mathrm{~g} / 1$ & 1,251 \\
\hline
\end{tabular}

O rendimento foi obtido colhendo-se $6 \mathrm{~m}$ lineares das três linhas centrais de cada parcela.

A população de plantas daninhas na área experimental foi estimada por ocasião da primeira avaliação de eficácia, contando-se as plantas contidas em quatro áreas de 0,25 $\mathrm{m}^{2}$, determinadas ao acaso dentro das parcelas testemunhas não capinadas.

\section{RESULTADOS E DISCUSSÃO}

\section{A. Controle de Plantas Daninhas}

A população de plantas daninhas nas áreas experimentais foi de 128 e 108 plantas $/ \mathrm{m}^{2}$ nas áreas de aplicação em pré e pós-emergência, respectivamente (Tabela 2). As principais espécies ocorrentes em ambas áreas foram a
Brachiaria decumbens, Richardia brasiliensis e Acanthospermum australe. A espécie Spermacoce latifolia também pode ser avaliada no experimento em pré-emergência em função de sua maior ocorrência na área.

A Richardia brasiliensis foi controlada de maneira excelente (superior a 90\%) ou total pelas misturas imazethapyr/imazapyr e imazapic/imazapyr em todas as doses utilizadas em pré-emergência (Tabela 3) nas três avaliações, exceto na dose mais baixa de imazethapyr/imazapyr $(79+26 \mathrm{~g} / \mathrm{ha})$, cuja eficácia de controle foi não aceitável para esta espécie. Estas mesmas misturas também apresentaram um controle excelente desta espécie quando aplicadas em pós-emergência (Tabela 4), porém apenas na avaliação realizada aos 30 dias após tratamento (DAT). Aos 60 DAT e na colheira, o controle resultante das aplicações em pós-emergência era apenas aceitável em algumas doses, sendo não aceitável em outras. Entretanto, a mistura imazapic/imazapyr, nas duas doses mais elevadas aplicadas em pós-emergência, 
Tabela 2. Composição do complexo florístico nas áreas experimentais. Fazenda Várzea, Planaltina-DF, 1998.

\begin{tabular}{|c|c|c|c|c|}
\hline \multirow{2}{*}{ Exp. } & \multirow{2}{*}{ Nome científico } & \multirow{2}{*}{ Nome comum } & \multicolumn{2}{|c|}{ Ocorrência } \\
\hline & & & (plantas $\left./ \mathrm{m}^{2}\right)$ & $(\%)$ \\
\hline \multirow{5}{*}{ Pré } & Acanthospermum australe & Carrapicho-rasteiro & 61 & 47,7 \\
\hline & Richardia brasiliensis & Poaia-branca & 24 & 18,7 \\
\hline & Brachiaria decumbens & Capim-braquiária & 23 & 18,0 \\
\hline & Spermacoce latitifolia & Erva-quente & 14 & 10,9 \\
\hline & Outras quatro espécies & - & 6 & 4,7 \\
\hline Total & & & 128 & 100,0 \\
\hline \multirow{6}{*}{ Pós } & Brachiaria decumbens & Capim-braquiária & 32 & 29,6 \\
\hline & Richardia brasiliensis & Poaia-branca & 26 & 24,1 \\
\hline & Acanthospermum australe & Carrapicho-rasteiro & 22 & 20,4 \\
\hline & Sida glaziovii & Guanxuma-branca & 10 & 9,3 \\
\hline & Commelina benghalensis & Trapoeraba & 8 & 7,4 \\
\hline & Outras sete espécies & - & 10 & 9,3 \\
\hline Total & & & 108 & 100,0 \\
\hline
\end{tabular}

Tabela 3. Efeito de misturas de herbicidas do grupo das imidazolinonas, aplicados em pré-emergência, no controle de plantas daninhas de folha-larga em híbrido de milho tolerante às imidazolinonas. Fazenda Várzea, PlanaltinaDF, $1997 / 98$.

\begin{tabular}{|c|c|c|c|c|c|c|c|}
\hline \multirow{3}{*}{ Tratamento } & \multirow{3}{*}{$\begin{array}{l}\text { Dose } \\
(\mathrm{g} / \mathrm{ha})\end{array}$} & \multicolumn{6}{|c|}{$\begin{array}{c}\text { Controle }(\%) \\
\text { Dias Após os Tratamentos }\end{array}$} \\
\hline & & 30 & 60 & $\mathrm{NC}$ & 30 & 60 & NC \\
\hline & & \multicolumn{3}{|c|}{ Acanthosperm um australe } & \multicolumn{3}{|c|}{ Richardia brasiliensis } \\
\hline Imazethapyr/imazapyr & $79+26$ & 77 & 84 & 88 & 78 & 78 & 76 \\
\hline Imazethapyr/imazapyr & $90+30$ & 95 & 93 & 97 & 95 & 98 & 95 \\
\hline Imazethapyr/imazapyr & $70+35$ & 82 & 79 & 76 & 93 & 91 & 94 \\
\hline Imazethapyr/imazapyr & $80+40$ & 98 & 91 & 94 & 90 & 95 & 92 \\
\hline Imazethapyr/imazapic & $70+35$ & 99 & 94 & 93 & 100 & 100 & 100 \\
\hline Imazethapyr/imazapic & $80+40$ & 98 & 91 & 95 & 90 & 100 & 98 \\
\hline Imazethapyr/imazapic & $60+30$ & 100 & 96 & 98 & 98 & 100 & 97 \\
\hline Imazapic/imazapyr & $70+35$ & 99 & 95 & 99 & 100 & 100 & 100 \\
\hline Imazapic/imazapyr & $68+22$ & 97 & 86 & 87 & 100 & 100 & 100 \\
\hline Imazapic/imazapyr & $79+26$ & 100 & 93 & 95 & 100 & 100 & 100 \\
\hline A trazine/metolachlor & 3250 & 100 & 93 & 97 & 98 & 100 & 100 \\
\hline Testemunha capinada & - & 100 & 100 & 100 & 100 & 100 & 100 \\
\hline Testemunha sem capina & - & 0 & 0 & 0 & 0 & 0 & 0 \\
\hline
\end{tabular}

também controlou de forma excelente a $R$. brasiliensis mesmo aos 60 DAT e na colheita. A mistura atrazine/metolachlor aplicada em pré-emergência apresentou controle total ou excelente desta espécie em todas as épocas de avaliação (Tabela 3). O herbicida nicosulfuron em pós-emergência controlou com eficácia aceitável a $R$. brasiliensis durante todo o ciclo do milho (Tabela 4).
O Acanthospermum australe, por ocasião da primeira avaliação de eficácia, foi controlado de maneira excelente pelas misturas de imidazolinonas aplicadas em pré-emergência ou pós-emergência, exceto pela dose mais baixa de imazethapyr/imazapyr em pré-emergência (Tabelas 3 e 4). As aplicações em pré-emergência também resultaram em controle excelente desta espécie aos $60 \mathrm{DAT}$ e por ocasião da 
Tabela 4. Efeito de misturas de herbicidas do grupo das imidazolinonas, aplicados em pós-emergência, no controle de plantas daninhas de folha-larga em híbrido de milho tolerante às imidazolinonas. Fazenda Várzea, PlanaltinaDF, $1997 / 98$.

\begin{tabular}{|c|c|c|c|c|c|c|c|}
\hline \multirow{3}{*}{ Tratamento } & \multirow{3}{*}{$\begin{array}{l}\text { Dose } \\
\text { (g/ha) }\end{array}$} & \multicolumn{6}{|c|}{$\begin{array}{c}\text { Controle }(\%) \\
\text { Dias Após os Tratamentos }\end{array}$} \\
\hline & & 30 & 60 & $\mathrm{NC}$ & 30 & 60 & $\mathrm{NC}$ \\
\hline & & \multicolumn{3}{|c|}{ Richardia brasiliensis } & \multicolumn{3}{|c|}{ Acanthospermum australe } \\
\hline Imazethapyr/imazapyr & $57+18$ & 93 & 77 & 79 & 95 & 81 & 85 \\
\hline Imazethapyr/imazapyr & $68+22$ & 93 & 80 & 82 & 93 & 90 & 91 \\
\hline Imazethapyr/imazapyr & $79+26$ & 94 & 83 & 83 & 94 & 90 & 92 \\
\hline Imazethapyr/imazapyr & $50+25$ & 91 & 71 & 77 & 91 & 73 & 79 \\
\hline Imazethapyr/imazapyr & $60+30$ & 90 & 83 & 85 & 94 & 85 & 82 \\
\hline Imazethapyr/imazapyr & $70+35$ & 91 & 80 & 85 & 91 & 90 & 90 \\
\hline Imazapic/imazapyr & $40+20$ & 93 & 80 & 82 & 93 & 78 & 79 \\
\hline Imazapic/imazapyr & $50+25$ & 94 & 89 & 86 & 94 & 84 & 88 \\
\hline Imazapic/imazapyr & $60+30$ & 94 & 93 & 94 & 94 & 83 & 88 \\
\hline Imazapic/imazapyr & $45+15$ & 94 & 88 & 85 & 94 & 86 & 87 \\
\hline Imazapic/imazapyr & $57+18$ & 93 & 84 & 85 & 93 & 84 & 82 \\
\hline Imazapic/imazapyr & $68+22$ & 94 & 93 & 92 & 94 & 91 & 94 \\
\hline Nicosulfuron & 60 & 88 & 85 & 86 & 87 & 80 & 85 \\
\hline Testemunha capinada & - & 100 & 100 & 100 & 100 & 100 & 100 \\
\hline Testemunha sem capina & - & 0 & 0 & 0 & 0 & 0 & 0 \\
\hline
\end{tabular}

$\mathrm{NC}=\mathrm{Na}$ Colheita.

Tabela 5. Efeito de misturas de herbicidas do grupo das imidazolinonas, aplicados em pré-emergência, no controle de Spermacoce latifolia e Brachiaria decumbens em híbrido de milho tolerante às imidazolinonas. Fazenda Várzea, Planaltina - DF, 1997/98.

\begin{tabular}{lcrrrrrr}
\hline & & \multicolumn{7}{c}{$\begin{array}{c}\text { Controle (\%) } \\
\text { Dias Após os Tratamentos }\end{array}$} \\
\cline { 3 - 8 } Tratamento & Dose $\mathbf{( g / h a )}$ & $\mathbf{3 0}$ & $\mathbf{6 0}$ & $\mathbf{N C}$ & $\mathbf{3 0}$ & $\mathbf{6 0}$ & NC \\
\cline { 3 - 8 } & & \multicolumn{7}{c}{ Spermacoce } & latifolia & Brachiaria decumbens \\
\hline Imazethapyr/imazapyr & $79+26$ & 80 & 90 & 88 & 47 & 49 & 57 \\
Imazethapyr/imazapyr & $90+30$ & 98 & 96 & 93 & 49 & 84 & 81 \\
Imazethapyr/imazapyr & $70+35$ & 85 & 93 & 91 & 57 & 49 & 51 \\
Imazethapyr/imazapyr & $80+40$ & 100 & 100 & 100 & 60 & 85 & 82 \\
Imazethapyr/imazapic & $70+35$ & 100 & 96 & 95 & 65 & 76 & 70 \\
Imazethapyr/imazapic & $80+40$ & 100 & 100 & 100 & 63 & 93 & 91 \\
Imazethapyr/imazapic & $60+30$ & 100 & 100 & 100 & 50 & 60 & 63 \\
Imazapic/imazapyr & $70+35$ & 100 & 99 & 100 & 51 & 77 & 75 \\
Imazapic/imazapyr & $68+22$ & 100 & 99 & 99 & 43 & 61 & 66 \\
Imazapic/imazapyr & $79+26$ & 100 & 98 & 100 & 45 & 75 & 77 \\
Atrazine/metolachlor & 3250 & 100 & 96 & 95 & 91 & 96 & 94 \\
Testemunha capinada & - & 100 & 100 & 100 & 100 & 100 & 100 \\
Testemunha sem capina & - & 0 & 0 & 0 & 0 & 0 & 0 \\
\hline
\end{tabular}

$\mathrm{NC}=$ Avaliação no dia da colheita da soja 
Tabela 6. Efeito de misturas de herbicidas do grupo das imidazolinonas, aplicados em pós-emergência, no controle de Brachiaria decumbens em híbrido de milho tolerante às imidazolinonas. Fazenda Várzea, Planaltina - DF, $1997 / 98$.

\begin{tabular}{|c|c|c|c|c|}
\hline \multirow{3}{*}{ Tratamento } & \multirow{3}{*}{$\begin{array}{c}\text { Dose } \\
\text { (g/ha) }\end{array}$} & \multicolumn{3}{|c|}{ Controle $(\%)$} \\
\hline & & \multicolumn{3}{|c|}{ Dias Após os Tratamentos } \\
\hline & & 30 & 60 & NC \\
\hline Imazethapyr/imazapyr & $57+18$ & 45 & 40 & 41 \\
\hline Imazethapyr/imazapyr & $68+22$ & 65 & 50 & 53 \\
\hline Imazethapyr/imazapyr & $79+26$ & 70 & 60 & 63 \\
\hline Imazethapyr/imazapyr & $50+25$ & 55 & 45 & 42 \\
\hline Imazethapyr/imazapyr & $60+30$ & 70 & 65 & 63 \\
\hline Imazethapyr/imazapyr & $70+35$ & 80 & 82 & 81 \\
\hline Imazapic/imazapyr & $40+20$ & 40 & 45 & 46 \\
\hline Imazapic/imazapyr & $50+25$ & 53 & 48 & 45 \\
\hline Imazapic/imazapyr & $60+30$ & 70 & 72 & 73 \\
\hline Imazapic/imazapyr & $45+15$ & 63 & 55 & 56 \\
\hline Imazapic/imazapyr & $57+18$ & 60 & 52 & 51 \\
\hline Imazapic/imazapyr & $68+22$ & 67 & 65 & 61 \\
\hline Nicosulfuron & 60 & 86 & 88 & 83 \\
\hline Testemunha capinada & - & 100 & 100 & 100 \\
\hline Testemunha sem capina & - & 0 & 0 & 0 \\
\hline
\end{tabular}

$\mathrm{NC}=$ Avaliação no dia da colheita da soja

colheita, exceto para as doses mais baixas da mistura imazethapyr/imazapyr e imazapic/imazapyr. No experimento em pós-emergência o controle permaneceu excelente com o imazethapyr/imazapyr a $68+22,79+26$ e $70+35 \mathrm{~g} / \mathrm{ha} \mathrm{e}$ com a mistura imazapic/imazapyr a $68+22 \mathrm{~g} / \mathrm{ha}$. Somente a dose mais baixa das misturas imazethapyr/imazapyr e imazapic/imazapyr em pós-emergência, ambas na proporção 2:1, apresentaram eficácia não aceitável de controle. Todas as demais doses, nas duas proporções, controlaram o A. australe com eficácia aceitável nas avaliações de 60 DAT e na colheita. A mistura atrazine/metolachlor controlou de forma excelente esta espécie em todas as avaliações, quando aplicada em pré-emergência. O nicosulfuron, em pós-emergência, controlou esta planta daninha com eficácia aceitável durante todo o ciclo do milho (Tabela 4).

A Brachiaria decumbens somente foi bem controlada em pré-emergência pelas misturas imazethapyr/imazapyr, nas doses de $90+30$ e $80+40 \mathrm{~g} /$ ha (controle aceitável) e imazethapyr/imazapic na dose de $80+40 \mathrm{~g} / \mathrm{ha}$, com excelente controle (Tabela 5). Nas aplicações de pós-emergência foi controlada com eficácia aceitável, durante todo o ciclo do milho, pela mistura imazethapyr/imazapyr a $70+35 \mathrm{~g} / \mathrm{ha} \mathrm{e}$ pelo nicosulfuron a $50 \mathrm{~g} / \mathrm{ha}$ (Tabela 6). Todos os demais tratamentos com herbicidas foram ineficientes no controle de B. decumbens.
A espécie Spermacoce latifolia só pôde ser avaliada no experimento de pré-emergência, sendo controlada de forma excelente por todas as misturas, à exceção do imazethapyr/ imazapyr na dose mais baixa $(79+26 \mathrm{~g} / \mathrm{ha})$ (Tabela 5).

\section{B. Fitotoxicidade}

Nenhum dos tratamentos, tanto nas aplicações em pré como em pós-emergência, foi tóxico às plantas de milho nas avaliações aos 30 e 60 DAT, confirmando que o híbrido é realmente tolerante aos herbicidas do grupo das imidazolinonas.

\section{Rendimento dos Grãos}

Todos os tratamentos para o controle das plantas daninhas, em ambos os experimentos apresentaram produtividades equivalentes entre si, porém superior à testemunha não capinada. O baixo rendimento do milho deve ter sido ocasionado pela baixa umidade do solo no momento da semeadura e também pela inadequação do híbrido às condições ambientais do local onde foram realizados os experimentos (Tabela 7). 
Tabela 7. Efeito de misturas de herbicidas aplicadas em pré ou pós-emergência na produtividade de grãos na cultura de milho tolerante às imidazolinonas. Fazenda Várzea, Planaltina-DF, 1997/98.

\begin{tabular}{|c|c|c|c|c|c|}
\hline \multicolumn{3}{|c|}{ Pré-emergência } & \multicolumn{3}{|c|}{ Pós-emergência } \\
\hline Tratamento & $\begin{array}{l}\text { Dose } \\
\text { (g/ha) }\end{array}$ & $\begin{array}{c}\text { Rendimento } \\
\text { (kg/ha) }\end{array}$ & Tratamento & $\begin{array}{l}\text { Dose } \\
\text { (g/ha) }\end{array}$ & $\begin{array}{c}\text { Rendimento } \\
(\mathrm{kg} / \mathrm{ha})\end{array}$ \\
\hline Imazethapyr/imazapyr & $79+26$ & $2940 \mathrm{a}$ & Imazethapyr/imazapyr & $57+18$ & $2810 \mathrm{a}^{1}$ \\
\hline Imazethapyr/imazapyr & $90+30$ & $2860 \mathrm{a}$ & Imazethapyr/imazapyr & $68+22$ & $2755 \mathrm{a}$ \\
\hline Imazethapyr/imazapyr & $70+35$ & $2955 \mathrm{a}$ & Imazethapyr/imazapyr & $79+26$ & $2920 \mathrm{a}$ \\
\hline Imazethapyr/imazapyr & $80+40$ & $2770 \mathrm{a}$ & Imazethapyr/imazapyr & $50+25$ & $2840 \mathrm{a}$ \\
\hline Imazethapyr/imazapic & $70+35$ & $2780 \mathrm{a}$ & Imazethapyr/imazapyr & $60+30$ & $2935 \mathrm{a}$ \\
\hline Imazethapyr/imazapic & $80+40$ & $2865 \mathrm{a}$ & Imazethapyr/imazapyr & $70+35$ & $2790 \mathrm{a}$ \\
\hline Imazethapyr/imazapic & $60+30$ & $2915 \mathrm{a}$ & Imazapic/imazapyr & $40+20$ & $2750 \mathrm{a}$ \\
\hline Imazapic/imazapyr & $70+35$ & $2875 \mathrm{a}$ & Imazapic/imazapyr & $50+25$ & $2855 \mathrm{a}$ \\
\hline Imazapic/imazapyr & $68+22$ & $2960 a$ & Imazapic/imazapyr & $60+30$ & $2905 \mathrm{a}$ \\
\hline Imazapic/imazapyr & $79+26$ & $2975 \mathrm{a}$ & Imazapic/imazapyr & $45+15$ & $2865 \mathrm{a}$ \\
\hline Atrazine/metolachlor & 3250 & $2970 \mathrm{a}$ & Imazapic/imazapyr & $57+18$ & $2975 \mathrm{a}$ \\
\hline Test. capinada & - & $3150 \mathrm{a}$ & Imazapic/imazapyr & $68+22$ & $2980 \mathrm{a}$ \\
\hline \multirow[t]{3}{*}{ Test. sem capina } & - & $1750 \mathrm{~b}$ & Nicosulfuron & 60 & $2965 \mathrm{a}$ \\
\hline & & & Test. capinada & - & $3050 \mathrm{a}$ \\
\hline & & & Test. sem capina & - & $1810 \mathrm{~b}$ \\
\hline
\end{tabular}

${ }^{1}$ Médias seguidas pela mesma letra não diferem entre si, de acordo com o teste de Duncan, ao nível de $5 \%$ de probabilidade.

\section{CONCLUSÕES}

A Richardia brasiliensis pode ser controlada de forma excelente (superior a 90\%) ou total com a utilização das seguintes misturas em pré-emergência: imazethapyr/imazapyr $(90+30,70+35,80+40 \mathrm{~g} / \mathrm{ha})$ imazethapyr/imazapic $(70+$ 35 e $80+40 \mathrm{~g} / \mathrm{ha})$ e imazapic/imazapyr $(60+30,70+35$, $68+22$ e $79+26 \mathrm{~g} / \mathrm{ha})$. A mistura imazethapyr/imazapyr é menos eficiente quando aplicada em pós-emergência nesta espécie. Aplicações em pós-emergência inicial de imazapic/ imazapyr $(60+30$ e $68+22 \mathrm{~g} / \mathrm{ha})$ também controlam de forma excelente esta espécie.

O Acanthospermum australe pode ser controlado de maneira excelente ou total pelas misturas imazethapyr/ imazapyr $(90+30$ e $80+40 \mathrm{~g} / \mathrm{ha})$, imazethapyr/imazapic $(70+35$ e $80+40)$ e imazapic/imazapyr $(60+30,70+35$, $68+22$ e $79+26 \mathrm{~g} / \mathrm{ha}$ ) em pré-emergência. Esta espécie também pode ser controlada de forma excelente com aplicações em pós-emergência das misturas imazethapyr/imazapyr $(68+22,79+26$ e $70+35 \mathrm{~g} / \mathrm{ha})$ e imazapic/imazapyr $(68+$ $22 \mathrm{~g} / \mathrm{ha})$.

A Brachiaria decumbens só é controlada de forma excelente, através do uso de imidazolinonas, pela mistura imazethapyr/imazapic na dose de $80+40 \mathrm{~g} / \mathrm{ha}$ em pré-emer- gência, o que é semelhante ao controle obtido com atrazine/ metolachlor. O controle desta espécie é aceitável pela mistura imazethapyr/imazapyr, nas doses de $90+30$ e $80+40 \mathrm{~g} /$ ha. Nas aplicações de pós-emergência esta espécie foi controlada com eficácia aceitável, durante todo o ciclo do milho, pela mistura imazethapyr/imazapyr a $70+35 \mathrm{~g} / \mathrm{ha}$ e pelo nicosulfuron a $50 \mathrm{~g} / \mathrm{ha}$. Todos os demais tratamentos com herbicidas foram ineficientes no controle de $B$. decumbens.

A espécie Spermacoce latifolia pode ser controlada de forma excelente por todas as misturas e doses utilizadas em pré-emergência, à exceção do imazethapyr/imazapyr na dose mais baixa $(79+26 \mathrm{~g} / \mathrm{ha})$.

Nenhum tratamento herbicida causou fitotoxicidade ao milho híbrido IMI-Corn nas doses utilizadas.

\section{LITERATURA CITADA}

AMERICAN CYANAMID COMPANY. Lightning herbicide: for use in IMI-Corn only. Lightning Specimen Label, PE - 5504. 1997. 18p. 
AUSTRALIAN WEEDS COMMITTEE. Guidelines for field evaluation of herbicides. Canberra Australian Government Publishing Service, 1979, p. A1-5.

EMBRAPA. Recomendações técnicas para a cultura da soja na região central do Brasil 1998/99. Londrina: 1998. 182p. (EMBRAPA-CNPSo. Documentos, 120).
FRANS. R. E.; TALBERT, R. E. Design of field experiments and the measurement and analysis of plant responses. In: TRUELOVE, B., Ed. Research Methods in Weed Science. 2a. ed., cap. 2. 1977.

LOUX, M.M.; STACHLER, J.M.; HARRISON, S.K. Weed control guide for Ohio field crops. IMI-Corn: postemergence herbicides. Ohio State University Extension Bulletin, Department of Horticulture and Crop Science. Bulletim 789. 1997. 4p. 